\title{
DISTRIBUTION OF UNITS OF REAL QUADRATIC NUMBER FIELDS
}

\author{
YEN-MEI J. CHEN, YOSHIYUKI KITAOKA AND JING YU1
}

\author{
Dedicated to the seventieth birthday of Professor Tomio Kubota
}

\begin{abstract}
Let $k$ be a real quadratic field and $\mathfrak{o}_{k}, E$ the ring of integers and the group of units in $k$. Denoting by $E(\mathfrak{p})$ the subgroup represented by $E$ of $\left(\mathfrak{o}_{k} / \mathfrak{p}\right)^{\times}$for a prime ideal $\mathfrak{p}$, we show that prime ideals $\mathfrak{p}$ for which the order of $E(\mathfrak{p})$ is theoretically maximal have a positive density under the Generalized Riemann Hypothesis.
\end{abstract}

\section{$\S 1$. Statement of the result}

Let $k$ be a real quadratic number field with discriminant $D_{0}$ and fundamental unit $\epsilon(>1)$, and let $\mathfrak{o}_{k}$ and $E$ be the ring of integers in $k$ and the set of units in $k$, respectively. For a prime ideal $\mathfrak{p}$ of $k$ we denote by $E(\mathfrak{p})$ the subgroup of the unit group $\left(\mathfrak{o}_{k} / \mathfrak{p}\right)^{\times}$of the residue class group modulo $\mathfrak{p}$ consisting of classes represented by elements of $E$ and set $I_{p}:=\left[\left(\mathfrak{o}_{k} / \mathfrak{p}\right)^{\times}: E(\mathfrak{p})\right]$, where $p$ is the rational prime lying below $\mathfrak{p}$. It is obvious that $I_{p}$ is independent of the choice of prime ideals lying above $p$. Set

$$
\ell_{p}:= \begin{cases}1, & \text { if } p \text { is decomposable or ramified in } k \\ p-1, & \text { if } p \text { remains prime in } k \text { and } N_{k / \mathbf{Q}}(\epsilon)=1 \\ (p-1) / 2, & \text { if } p \text { remains prime in } k \text { and } N_{k / \mathbf{Q}}(\epsilon)=-1\end{cases}
$$

where $N_{k / \mathbf{Q}}$ stands for the norm from $k$ to the rational number field $\mathbf{Q}$. In $[\mathrm{IK}]$ we have shown that $\ell_{p}$ divides $I_{p}$ and we observed that in each case the set of prime numbers satisfying $I_{p}=\ell_{p}$ has a natural density. K. Masima found that the values in tables there, are connected with the Artin constant $A:=\prod_{p}\left(1-\frac{1}{p(p-1)}\right)=0.3739558 \cdots$ and showed in $[\mathrm{M}]$ that the set of decomposable prime numbers satisfying $I_{p}=\ell_{p}$ has a density under the

Received October 7, 1999.

1991 Mathematics Subject Classification: 11R45

${ }^{1}$ Research partially supported by National Science Council, Rep. of China. 
Generalized Riemann Hypothesis (GRH) following [H]. In this paper, we treat the case where prime numbers remain prime in $k$ following $[\mathrm{H}],[\mathrm{M}]$. However, instead of counting prime ideals which are completely decomposable, we use the Chebotarev Density Theorem by [LO], [S] under the GRH. Our main result is the following.

TheOREM. Let $\mathbb{P}(x)$ be the set of odd prime numbers $p \leq x$ which remain prime in $k$ and let $N(x)$ be the subset of $p \in \mathbb{P}(x)$ satisfying $I_{p}=\ell_{p}$. Then we have

$$
\sharp N(x)=c_{0} \operatorname{Li}(x)+O\left(x \log \log x /(\log x)^{2}\right)
$$

for a positive constant $c_{0}$ under the GRH.

Here fields where the GRH is involved are $k\left(\zeta_{2 n}, \sqrt[t]{\epsilon}\right)$ for square-free natural numbers $n$ and $t=n$ or $2 n$, where $\zeta_{m}$ stands for a primitive $m$-th root of unity. The function $\operatorname{Li}(x)$ stands for $\int_{2}^{x} d t / \log t$ as usual.

\section{$\S 2$. Algebraic preparation}

Throughout this paper, we keep the notation in Section 1. The main results in this section are Theorems 1 and 2 .

LEMMA 1. Let $n$ be a square-free integer $(\geq 1)$ and suppose that $k \not \subset$ $\mathbf{Q}\left(\zeta_{2 n}\right)$ and suppose $\sqrt[2 n]{\epsilon} \in \mathbf{R}$. Set

$$
K:= \begin{cases}k\left(\zeta_{2 n}, \sqrt[2 n]{\epsilon}\right), & \text { if } N_{k / \mathbf{Q}}(\epsilon)=1, \\ k\left(\zeta_{2 n}, \sqrt[n]{\epsilon}\right), & \text { if } N_{k / \mathbf{Q}}(\epsilon)=-1\end{cases}
$$

and let $N:=\left[K: k\left(\zeta_{2 n}\right)\right]$ be the extension degree of fields. Then we have

$$
\begin{aligned}
N & =[K: \mathbf{Q}] / 2 \varphi(2 n) \\
& = \begin{cases}n, & \text { either if } N_{k / \mathbf{Q}}(\epsilon)=1 \text { and } \sqrt{\epsilon} \in k\left(\zeta_{2 n}\right), \\
2 n, & \text { if } N_{k / \mathbf{Q}}(\epsilon)=1 \text { and } \sqrt{\epsilon} \notin k\left(\zeta_{2 n}\right),\end{cases}
\end{aligned}
$$

where $\varphi(m)$ is the Euler function.

Proof. Let us recall that

for an integer $m$, and an element $a$ in a field $F(\operatorname{ch}(F) \neq 2)$ which is not contained in $F^{p}$ for every prime divisor $p$ of $m$, a polynomial $x^{m}-a$ is irreducible either if $4 \nmid m$, or if $4 \mid m$ and $-4 a \notin F^{4}$. 
Let $q$ be an odd prime or 4 and suppose $q \mid 2 n$. We show $\sqrt[q]{\epsilon} \notin k\left(\zeta_{2 n}\right)$ first. Suppose $\sqrt[q]{\epsilon} \in k\left(\zeta_{2 n}\right)$; then $\mathbf{Q}(\sqrt[q]{\epsilon})=\mathbf{Q}\left((\sqrt[2 n]{\epsilon})^{2 n / q}\right) \subset \mathbf{R}$ is a subfield of an abelian field $k\left(\zeta_{2 n}\right)$. Hence any conjugate element of $\sqrt[q]{\epsilon}$ should be real. This is a contradiction. Hence $\sqrt[q]{\epsilon}$ is not in $k\left(\zeta_{2 n}\right)$.

Let us show that $x^{2 n}-\epsilon$ is irreducible over $k\left(\zeta_{2 n}\right)$ if $\sqrt{\epsilon} \notin k\left(\zeta_{2 n}\right)$. We have only to consider the case of $2 \mid n$. Suppose that $2 \mid n, \sqrt[4]{-4 \epsilon} \in k\left(\zeta_{2 n}\right)$ and $\sqrt{\epsilon} \notin k\left(\zeta_{2 n}\right)$; then $\sqrt{-4 \epsilon} \in k\left(\zeta_{2 n}\right)$ and $\sqrt{-1} \sqrt{\epsilon} \in k\left(\zeta_{2 n}\right)$ hold. This contradicts that $\sqrt{\epsilon} \notin k\left(\zeta_{2 n}\right)$ since $\sqrt{-1} \in k\left(\zeta_{2 n}\right)$ holds by $2 \mid n$. Thus the above criterion yields that a polynomial $x^{2 n}-\epsilon$ is irreducible over $k\left(\zeta_{2 n}\right)$ if $\sqrt{\epsilon} \notin k\left(\zeta_{2 n}\right)$. Then we have $N=\left[k\left(\zeta_{2 n}, \sqrt[2 n]{\epsilon}\right): k\left(\zeta_{2 n}\right)\right]=2 n$. If, next $\sqrt{\epsilon} \in k\left(\zeta_{2 n}\right)$, then a polynomial $x^{n}-\sqrt{\epsilon}$ is irreducible over $k\left(\zeta_{2 n}\right)$ and then we have $N=\left[k\left(\zeta_{2 n},(\sqrt{\epsilon})^{1 / n}\right): k\left(\zeta_{2 n}\right)\right]=n$. Similarly, $x^{n}-\epsilon$ is irreducible over $k\left(\zeta_{2 n}\right)$ if $N_{k / \mathbf{Q}}(\epsilon)=-1$ and $2 \nmid n$.

Remark. In Lemma 1, a rational prime $p$ is unramified in $K$ if $p \nmid 2 n D_{0}$.

Proposition 1. Let $n, K, N$ be those in Lemma 1. Let $\eta \in$ $\operatorname{Gal}\left(k\left(\zeta_{2 n}\right) / \mathbf{Q}\right)$ be an automorphism such that $\eta\left(\zeta_{2 n}\right)=\zeta_{2 n}^{-1}$ and $\eta$ induces the non-trivial automorphism of $\operatorname{Gal}(k / \mathbf{Q})$.

(I) The case of $N_{k / \mathbf{Q}}(\epsilon)=1$. There exists an automorphism $\rho$ of order 2 in $\operatorname{Gal}(K / \mathbf{Q})$ such that $\rho=\eta$ on $k\left(\zeta_{2 n}\right)$ if and only if (i) $N=2 n$, (ii) $N=n$ is odd, or (iii) $N=n$ is even and $\eta(\sqrt{\epsilon}) \sqrt{\epsilon}=1$. When $\rho$ exists, it is in the center of $\operatorname{Gal}(K / \mathbf{Q})$ and satisfies $\rho(\sqrt[2 n]{\epsilon})= \pm \sqrt[2 n]{\epsilon}-1$ and both signs \pm are possible if and only if $N$ is even.

(II) The case of $N_{k / \mathbf{Q}}(\epsilon)=-1$. If $n$ is odd, then there exists a unique automorphism $\rho$ of order 2 in $\operatorname{Gal}(K / \mathbf{Q})$ such that $\rho=\eta$ on $k\left(\zeta_{2 n}\right)$. It is in the center of $\operatorname{Gal}(K / \mathbf{Q})$ and $\rho(\sqrt[n]{\epsilon})=-\sqrt[n]{\epsilon}-1$. If $n$ is even, then there is no such automorphism.

Proof. Set $t=2 n$ or $n$ according to $N_{k / \mathbf{Q}}(\epsilon)=1$ or -1 , respectively. If $\rho \in \operatorname{Gal}(K / \mathbf{Q})$ is an extension of $\eta$ and $\rho^{2}=\mathrm{id}$, then setting $\rho(\sqrt[t]{\epsilon})=$ $\delta \sqrt[t]{\epsilon}-1$ for some $\delta \in K$, we have $\delta^{2 n}=1$ and hence $\delta$ is a $2 n$-th root of unity and hence $\rho(\delta)=\eta(\delta)=\delta^{-1}$ and $\sqrt[t]{\epsilon}=\rho^{2}(\sqrt[t]{\epsilon})=\rho(\delta \sqrt[t]{\epsilon}-1)=$ $\delta^{-1}(\delta \sqrt[t]{\epsilon}-1)^{-1}=\delta^{-2} \sqrt[t]{\epsilon}$. Thus we have $\delta= \pm 1$.

Proof of the case (I).

Suppose $N_{k / \mathbf{Q}}(\epsilon)=1$. Assume that either $N=2 n$, or $N=n$ is odd, first. We define $\xi_{n}= \pm 1$ by

$$
\xi_{n}:= \begin{cases}1, & \text { if } N=2 n \\ \eta(\sqrt{\epsilon}) \sqrt{\epsilon}, & \text { if } N=n\end{cases}
$$


where if $N=n, \sqrt{\epsilon} \in k\left(\zeta_{2 n}\right)$ holds by virtue of Lemma 1 , and $\eta$ can act on $\sqrt{\epsilon}$.

Let $\eta^{\prime} \in \operatorname{Gal}(K / \mathbf{Q})$ be an extension of $\eta \in \operatorname{Gal}\left(k\left(\zeta_{2 n}\right) / \mathbf{Q}\right)$; then we have

$$
\left(\eta^{\prime}(\sqrt[2 n]{\epsilon}) \sqrt[2 n]{\epsilon} \xi_{n}\right)^{N}=\eta(\epsilon) \epsilon=1
$$

and hence $\eta^{\prime}(\sqrt[2 n]{\epsilon})=\zeta_{N}^{r} \xi_{n} \sqrt[2 n]{\epsilon}{ }^{-1}$ for some integer $r$ and a primitive $N$-th root $\zeta_{N}$ of unity. Since $K=k\left(\zeta_{2 n}\right)(\sqrt[2 n]{\epsilon})$ and $N=\left[K: k\left(\zeta_{2 n}\right)\right]$, there exists an automorphism $\alpha \in \operatorname{Gal}\left(K / k\left(\zeta_{2 n}\right)\right)$ such that $\alpha(\sqrt[2 n]{\epsilon})=\zeta_{N}^{r} \sqrt[2 n]{\epsilon}$. Thus an automorphism $\rho:=\alpha \eta^{\prime}$ is an extension of $\eta$ and satisfies $\rho(\sqrt[2 n]{\epsilon})=\xi_{n} \sqrt[2 n]{\epsilon}{ }^{-1}$ and the order of $\rho$ is equal to 2 .

Secondly, we consider the case where $N=n$ is even. By Lemma 1, we have $\sqrt{\epsilon} \in k\left(\zeta_{2 n}\right)$. Take any extension $\eta^{\prime}$ in $\operatorname{Gal}(K / \mathbf{Q})$ of $\eta$. Since $\left(\eta^{\prime}(\sqrt[2 n]{\epsilon}) \sqrt[2 n]{\epsilon}\right)^{2 n}=\eta(\epsilon) \epsilon=1$, we have $\eta^{\prime}\left((\sqrt{\epsilon})^{1 / n}\right)=\zeta_{2 n}^{t}(\sqrt{\epsilon})^{-1 / n}$ for some integer $t$, and

$$
\eta(\sqrt{\epsilon})=\zeta_{2 n}^{t n} \sqrt{\epsilon}^{-1}
$$

If, hence $\eta(\sqrt{\epsilon}) \sqrt{\epsilon}=1$, then $t$ is even and since $\left[K: k\left(\zeta_{2 n}\right)\right]=n$ and $\sqrt{\epsilon} \in k\left(\zeta_{2 n}\right)$, we can take $\alpha \in \operatorname{Gal}\left(K / k\left(\zeta_{2 n}\right)\right)$ so that $\alpha(\sqrt[2 n]{\epsilon})=\zeta_{2 n}^{t} \sqrt[2 n]{\epsilon}$, and therefore $\rho:=\alpha \eta^{\prime}$ is what we want. If $\eta(\sqrt{\epsilon}) \sqrt{\epsilon}=-1$, then $t$ is odd and the order of $\eta^{\prime}$ is not equal 2 , since $\eta^{\prime 2}(\sqrt[2 n]{\epsilon})=\zeta_{n}^{-t} \sqrt[2 n]{\epsilon} \neq \sqrt[2 n]{\epsilon}$. Thus we have completed the proof of the first assertion. Next, we show that if $\rho_{ \pm} \in \operatorname{Gal}(K / \mathbf{Q})$ is an extension of $\eta$ such that $\rho_{ \pm}(\sqrt[2 n]{\epsilon})= \pm \sqrt[2 n]{\epsilon}{ }^{-1}$, then $\rho_{ \pm}$is in the center of $\operatorname{Gal}(K / \mathbf{Q})$. Take an element $u \in \operatorname{Gal}(K / \mathbf{Q})$; then $u(\sqrt[2 n]{\epsilon})=\zeta_{2 n}^{r} \sqrt[2 n]{\epsilon}$ or $\zeta_{2 n}^{r} \sqrt[2 n]{\epsilon}-1$ for some integer $r$. If $u(\sqrt[2 n]{\epsilon})=\zeta_{2 n}^{r} \sqrt[2 n]{\epsilon}$, then $\rho_{ \pm} u(\sqrt[2 n]{\epsilon})= \pm \zeta_{2 n}^{-r} \sqrt[2 n]{\epsilon}-1$ and $u \rho_{ \pm}(\sqrt[2 n]{\epsilon})=u( \pm \sqrt[2 n]{\epsilon}-1)= \pm \zeta_{2 n}^{-r} \sqrt[2 n]{\epsilon}-1$ and hence $\rho_{ \pm} u=u \rho_{ \pm}$. The case of $u(\sqrt[2 n]{\epsilon})=\zeta_{2 n}^{r} \sqrt[2 n]{\epsilon}-1$ is similar and then $\rho_{ \pm}$ is in the center of $\operatorname{Gal}(K / \mathbf{Q})$. Lastly, suppose that $N$ is even; then there is an automorphism $\kappa \in \operatorname{Gal}\left(K / k\left(\zeta_{2 n}\right)\right)$ such that $\kappa(\sqrt[2 n]{\epsilon})=-\sqrt[2 n]{\epsilon}$ and hence both signs \pm are possible. Conversely, if there exist automorphisms $\rho_{ \pm} \in \operatorname{Gal}(K / \mathbf{Q})$ such that $\rho_{ \pm}=\eta$ on $k\left(\zeta_{2 n}\right)$ and $\rho_{ \pm}(\sqrt[2 n]{\epsilon})= \pm \sqrt[2 n]{\epsilon}-1$, then $\rho_{-} \rho_{+}$is the identity on $k\left(\zeta_{2 n}\right)$ and $\rho_{-} \rho_{+}(\sqrt[2 n]{\epsilon})=-\sqrt[2 n]{\epsilon}$ and hence the order of $\rho_{-} \rho_{+} \in \operatorname{Gal}\left(K / k\left(\zeta_{2 n}\right)\right)$ is two and it yields that $N=\left[K: k\left(\zeta_{2 n}\right)\right]$ is even. Thus we have completed the proof of the case (I).

Proof of the case (II).

Suppose $N_{k / \mathbf{Q}}(\epsilon)=-1$. First we consider the case where $n$ is odd; Since $\eta(\epsilon)=-\epsilon^{-1}$, taking an extension $\eta^{\prime} \in \operatorname{Gal}(K / \mathbf{Q})$ of $\eta \in \operatorname{Gal}\left(k\left(\zeta_{2 n}\right) / \mathbf{Q}\right)$, we have $\eta^{\prime}(\sqrt[n]{\epsilon})=-\zeta_{n}^{r} \sqrt[n]{\epsilon}{ }^{-1}$ for some integer $r$. There exists an automorphism $\alpha \in \operatorname{Gal}\left(K / k\left(\zeta_{2 n}\right)\right)$ such that $\alpha(\sqrt[n]{\epsilon})=\zeta_{n}^{r} \sqrt[n]{\epsilon}$ since $\left[K: k\left(\zeta_{2 n}\right)\right]=n$ is odd. 
We have only to set $\rho:=\alpha \eta^{\prime} . \rho$ is in the center of $\operatorname{Gal}(K / \mathbf{Q})$ as above. If there are two automorphisms $\rho_{ \pm}$of order 2 such that $\rho_{ \pm}$is $\eta$ on $k\left(\zeta_{2 n}\right)$, then $\rho_{ \pm}(\sqrt[n]{\epsilon})= \pm \sqrt[n]{\epsilon}$ should hold as at the beginning of the proof. As in the proof of the case (I), it implies the extension degree $\left[K: k\left(\zeta_{2 n}\right)\right]=n$ is even, which is a contradiction. Lastly suppose that $n$ is even and there is an automorphism $\rho$ of order 2 in $\operatorname{Gal}(K / \mathbf{Q})$ such that $\rho=\eta$ on $k\left(\zeta_{2 n}\right)$; then $\rho(\epsilon)=-\epsilon^{-1}$ implies $\rho(\sqrt[n]{\epsilon})=\zeta_{2 n}^{r} \sqrt[n]{\epsilon}^{-1}$ for an odd integer $r$. Then $\sqrt[n]{\epsilon}=\rho^{2}(\sqrt[n]{\epsilon})=\zeta_{2 n}^{-r}\left(\zeta_{2 n}^{r} \sqrt[n]{\epsilon}-1\right)^{-1}=\zeta_{2 n}^{-2 r} \sqrt[n]{\epsilon}$ implies $\zeta_{n}^{r}=\zeta_{2 n}^{2 r}=1$, which is a contradiction, since $2 \mid n$ and $2 \nmid r$. This completes the proof of the proposition.

Lemma 2. Suppose $N_{k / \mathbf{Q}}(\epsilon)=1$ and let $p$ be an odd prime which remains in $k$. Then for a square-free natural number $n, n(p-1) \mid I_{p}$ holds if and only if $p+1 \equiv 0 \bmod 2 n$ and each prime ideal of $k\left(\zeta_{2 n}\right)$ lying above $p$ is completely decomposable at $K=k\left(\zeta_{2 n}\right)(\sqrt[2 n]{\epsilon})$.

Proof. First note that $p-1$ divides $I_{p}$ as in the introduction. Let us show the "only if" part. Suppose that $n(p-1) \mid I_{p}$ and set $t=I_{p} / n(p-1)$ $(\in \mathbf{Z})$. Since $p^{2}-1=\sharp(\mathfrak{o} /(p))^{\times}=I_{p} \cdot \sharp E((p))$, we have $(p+1) / n=$ $\left(p^{2}-1\right) / n(p-1)=I_{p} \cdot \sharp E((p)) / n(p-1)=t \sharp E((p)) \equiv 0 \bmod 2$ since $\sharp E((p)) \equiv 0 \bmod 2$ by $\pm 1 \in E((p))$. Hence we have $p+1 \equiv 0 \bmod 2 n$. Next we show the following

\section{Claim. The relative degree of $p$ at $k\left(\zeta_{2 n}\right) / \mathbf{Q}$ is 2 .}

Let $\mathfrak{p}$ be a prime ideal of $k\left(\zeta_{2 n}\right)$ lying above $p$. In the local field $k\left(\zeta_{2 n}\right)_{\mathfrak{p}}$, the closure of $k$ is an unramified extension of $\mathbf{Q}_{p}$ of degree 2 and $p \equiv$ $-1 \bmod 2 n$ implies the closure of $\mathbf{Q}\left(\zeta_{2 n}\right)$ has the same property, and the uniqueness of the unramified extension of degree 2 over $\mathbf{Q}_{p}$ implies that $k\left(\zeta_{2 n}\right)_{\mathfrak{p}}$ is the unramified extension of degree 2 over $\mathbf{Q}_{p}$. This completes the proof of the claim.

Let $\alpha \in \mathfrak{o}_{k}$ be a generator of $\left(\mathfrak{o}_{k} /(p)\right)^{\times}$and $r$ the order of $\epsilon$ in $\left(\mathfrak{o}_{k} /(p)\right)^{\times}$, and define an integer $u$ with $(u, r)=1$ by $\epsilon \equiv \alpha^{u\left(p^{2}-1\right) / r} \bmod (p)$. Since $p^{2}-1=I_{p} \cdot \sharp E((p)) \equiv 0 \bmod n(p-1) \cdot r \equiv 0 \bmod 2 n r$, we have $p^{2}-1=2 n r w$ for some integer $w$. Then $\epsilon \equiv\left(\alpha^{u w}\right)^{2 n} \bmod (p)$ implies that the equation $x^{2 n}=\epsilon$ has a solution in the local field $k_{(p)}$ by successive approximation by Newton. Let $\mathfrak{p}$ be a prime ideal of $k\left(\zeta_{2 n}\right)$ lying above $p$; then $k\left(\zeta_{2 n}\right)_{\mathfrak{p}} \cong k_{(p)}$ follows from the Claim and hence the equation $x^{2 n}=\epsilon$ has a solution in $k\left(\zeta_{2 n}\right)_{\mathfrak{p}}$, hence $\mathfrak{p}$ is completely decomposable in $k\left(\zeta_{2 n}, \sqrt[2 n]{\epsilon}\right)$. 
Next let us show the "if" part. Let $\mathfrak{P}, \mathfrak{p}\left(=\mathfrak{P} \cap k\left(\zeta_{2 n}\right)\right)$ be prime ideals of $k\left(\zeta_{2 n}, \sqrt[2 n]{\epsilon}\right), k\left(\zeta_{2 n}\right)$ lying above $p$, respectively. By the assumption, the relative degree of $\mathfrak{P} / \mathfrak{p}$ is one. Hence the equation $x^{2 n}-\epsilon=0$ is soluble in the local field $k\left(\zeta_{2 n}\right)_{\mathfrak{p}}$. Since $p+1 \equiv 0 \bmod 2 n, p$ is unramified in $\mathbf{Q}\left(\zeta_{2 n}\right)$ and the closure of $\mathbf{Q}\left(\zeta_{2 n}\right)$ in $k\left(\zeta_{2 n}\right)_{\mathfrak{p}}$ is $\mathbf{Q}_{p}$ or its unramified quadratic extension. Thus $k\left(\zeta_{2 n}\right)_{\mathfrak{p}}$ is the unramified quadratic extension of $\mathbf{Q}_{p}$ and hence $x^{2 n}=\epsilon$ is soluble in $k_{(p)} \cong k\left(\zeta_{2 n}\right)_{\mathfrak{p}}$. Hence there exist a primitive root $\alpha \in \mathfrak{o}_{k}$ and an integer $u \in \mathbf{Z}$ such that

$$
\left(\alpha^{u}\right)^{2 n} \equiv \epsilon \bmod (p)
$$

(i) The case where $E((p))=\langle \pm 1, \epsilon \bmod (p)\rangle$ but $E((p)) \neq\langle\epsilon \bmod (p)\rangle$. We note that the assumption yields $\epsilon^{t} \not \equiv-1 \bmod (p)$ for any integer $t$. Let $r$ be the order of $\epsilon \bmod (p)$ in $\left(\mathfrak{o}_{k} /(p)\right)^{\times}$. If $r$ is even, $\left(\epsilon^{r / 2}\right)^{2} \equiv$ $1 \bmod (p)$ and hence $\epsilon^{r / 2} \equiv \pm 1 \bmod (p)$. Since $r$ is the order of $\epsilon \bmod (p)$, we have $\epsilon^{r / 2} \equiv-1 \bmod (p)$. It contradicts the assumption. Hence $r$ is odd and $\sharp E((p))=2 r$ implies $p^{2}-1=I_{p} \cdot 2 r$. Now we have $1 \equiv \epsilon^{r} \equiv\left(\alpha^{u}\right)^{2 n r} \bmod (p)$ and then we have $2 n r u \equiv 0 \bmod p^{2}-1$ and $2 n t u \not \equiv 0 \bmod p^{2}-1$ for any proper divisor $t$ of $r$, since $r$ is the order of $\langle\epsilon \bmod (p)\rangle$. Set $2 n r u=w\left(p^{2}-1\right)$ for an integer $w$. Then we have

$$
(r, w)=1 \quad \text { and } \quad r u=w(p-1) \frac{p+1}{2 n}
$$

Let us show $(r, p-1)=1$. If a prime number $q$ divides $(r, p-1)$, then $q$ is odd, since $r$ is odd. On the other hand, $\mathbf{Z} \ni I_{p} /(p-1)=(p+1) / 2 r$ and $q \mid r$ imply $q \mid(p+1)$. Therefore $q$ divides $p \pm 1$ and hence $q=2$, which is the contradiction and hence $(r, p-1)=1$. Thus $r$ divides $(p+1) / 2 n$ and hence $I_{p} / n(p-1)=2 r I_{p} / 2 r n(p-1)=\left(p^{2}-1\right) / 2 r n(p-1)=(p+1) / 2 r n \in \mathbf{Z}$ which yields that $n(p-1)$ divides $I_{p}$.

(ii) The case where $E((p))=\langle\epsilon \bmod (p)\rangle$.

Since $1 \not \equiv-1 \bmod (p)$, the order $r$ of $\epsilon \bmod (p)$ in $\left(\mathfrak{o}_{k} /(p)\right)^{\times}$is even. As in the case $(\mathrm{i})$, we have $2 n u r \equiv 0 \bmod p^{2}-1$ and for any proper divisor $t$, we have 2 nut $\not \equiv 0 \bmod p^{2}-1$. Set $2 n u r=w\left(p^{2}-1\right)(w \in \mathbf{Z})$; then $(r, w)=1$ follows and if a prime number $q$ divides $(r, p-1)$, then $q \mid r$ and

$$
\frac{p+1}{r}=\frac{I_{p}}{p-1} \in \mathbf{Z}
$$


imply $q \mid(p+1)$ and hence $q=2$. Set $r=2^{t} \cdot r^{\prime}\left(r^{\prime}:\right.$ odd $)$; then we have shown $\left(r^{\prime}, p-1\right)=1$ and $2^{t} r^{\prime} u=w(p-1) \frac{p+1}{2 n}$ implies

$$
r^{\prime} \mid \frac{p+1}{2 n}
$$

by $(r, w)=1$ and $\left(r^{\prime}, p-1\right)=1$. Let us show $n r \mid(p+1)$. If $n$ is odd, then $(p+1) / r \in \mathbf{Z}$ implies $2^{t} \mid(p+1)$ and hence $(p+1) / 2 n r^{\prime} \in \mathbf{Z}$ implies $r=2^{t} r^{\prime} \mid \frac{p+1}{n}$. If $n$ is even, then $p+1 \equiv 0 \bmod 2 n \equiv 0 \bmod 4$, and hence $(p-1) / 2$ is odd. Since $2^{t} r^{\prime} u=w \frac{p-1}{2} \frac{p+1}{n}$ and $\left(r, w \frac{p-1}{2}\right)=1$, we have $r \mid \frac{p+1}{n}$. Thus we have $I_{p}=\left(p^{2}-1\right) / r=\frac{p+1}{n r} \cdot(p-1) n \equiv 0 \bmod (p-1) n$ and hence we have completed the proof.

THEOREM 1. Suppose that $N_{k / \mathbf{Q}}(\epsilon)=1$ and $p$ is an odd prime number which remains prime in $k$. Let $n$ be a square-free integer $(\geq 1)$. Then $n(p-$ 1) $\mid I_{p}$ holds if and only if $k \not \subset \mathbf{Q}\left(\zeta_{2 n}\right)$ and for a prime ideal $\mathfrak{P}$ of $K=$ $k\left(\zeta_{2 n}, \sqrt[2 n]{\epsilon}\right)$ lying above $p$, the Frobenius automorphism $\rho_{0}=\left(\frac{K / \mathbf{Q}}{\mathfrak{P}}\right)$ is equal to an automorphism $\rho$ given in Proposition 1.

Proof. Suppose $n(p-1) \mid I_{p}$; then $p+1 \equiv 0 \bmod 2 n$ follows from Lemma 2 and hence $\rho_{0}$ is the complex conjugation on $\mathbf{Q}\left(\zeta_{2 n}\right)$ and then $\rho_{0}$ fixes each element in $k$ if $k \subset \mathbf{Q}\left(\zeta_{2 n}\right)$. On the other hand, $p$ remains prime in $k$ by the assumption and hence $\rho_{0}$ is a non-trivial automorphism of $k$, which is a contradiction. Thus we have $k \not \subset \mathbf{Q}\left(\zeta_{2 n}\right)$. By Lemma 2 , the relative degree of $\mathfrak{P}$ is two and so $\rho_{0}^{2}=\mathrm{id}$. and hence we have $\rho_{0}=\rho$ given in Proposition 1.

Conversely suppose that $k \not \subset \mathbf{Q}\left(\zeta_{2 n}\right)$ and $\rho_{0}$ is an automorphism of order 2 and it is equal to $\eta$ in Proposition 1 on $k\left(\zeta_{2 n}\right)$. Then $\rho_{0}$ is the complex conjugation on $\mathbf{Q}\left(\zeta_{2 n}\right)$ and then $p+1 \equiv 0 \bmod 2 n$. Since the order of $\rho_{0}$ is two, we have $\left[K_{\mathfrak{P}}: \mathbf{Q}_{p}\right]=2$ and $\left[k_{(p)}: \mathbf{Q}_{p}\right]=2$, which yields that the relative degree of $\mathfrak{P}$ at $K / k$ and hence at $K / k\left(\zeta_{2 n}\right)$ is one. Now Lemma 2 implies $n(p-1) \mid I_{p}$.

Lemma 3. Suppose $N_{k / \mathbf{Q}}(\epsilon)=-1$ and let $p$ be an odd prime which remains prime in $k$. Then for a square-free natural number $n, n \frac{p-1}{2} \mid I_{p}$ holds if and only if $p+1 \equiv 0 \bmod 2 n$ and each prime ideal of $k\left(\zeta_{2 n}\right)$ lying above $p$ is completely decomposable at $K=k\left(\zeta_{2 n}\right)(\sqrt[n]{\epsilon})$.

Proof. We note that $\frac{p-1}{2} \mid I_{p}$ as in the introduction ([IK]). Set $r:=$ $\sharp E((p))$. We claim $r \equiv 0 \bmod 4$. Because of $\epsilon^{p+1} \equiv-1 \bmod (p)([\mathrm{IK}])$, 
$E((p))$ is generated by $\epsilon \bmod (p) . \pm 1 \in E((p))$ implies $r \equiv 0 \bmod 2$. Suppose that $r=2 t$ for some odd integer $t$; then we have $\epsilon^{(p+1) t} \equiv-1 \bmod (p)$ and on the other hand $(p+1) t \equiv 0 \bmod 2 t$ implies $\epsilon^{(p+1) t} \equiv 1 \bmod (p)$, which is a contradiction. Therefore $r \equiv 0 \bmod 4$ and note $p^{2}-1=I_{p} \cdot r$.

First suppose $n \frac{p-1}{2} \mid I_{p}$ and set $I_{p}=n \frac{p-1}{2} \cdot t$ for an integer $t$. Then we have $(p+1) / n=\left(p^{2}-1\right) / n(p-1)=I_{p} r / n(p-1)=\operatorname{tr} / 2 \in 2 \mathbf{Z}$ and then

$$
p+1 \equiv 0 \bmod 2 n .
$$

Since the order of $\epsilon$ in $\left(\mathfrak{o}_{k} /(p)\right)^{\times}$is $r$, we can take a generator $\alpha \in \mathfrak{o}_{k}$ of $\left(\mathfrak{o}_{k} /(p)\right)^{\times}$so that $\epsilon \equiv \alpha^{u\left(p^{2}-1\right) / r} \bmod (p)$ for an integer $u$ with $(u, r)=1$. Then we have $\epsilon \equiv\left(\alpha^{u\left(p^{2}-1\right) / n r}\right)^{n} \bmod (p)$, where $\left(p^{2}-1\right) / n r=I_{p} / n$ is an integer. Hence $x^{n} \equiv \epsilon \bmod (p)$ is soluble in $\mathfrak{o}_{k}$ and $x^{n}=\epsilon$ has a solution in the local field $k_{(p)}$. Let $\mathfrak{P}$ be a prime ideal of $K=k\left(\zeta_{2 n}, \sqrt[n]{\epsilon}\right)$ lying above $p$ and set $\mathfrak{p}=\mathfrak{P} \cap k\left(\zeta_{2 n}\right)$; then $p+1 \equiv 0 \bmod 2 n$ implies that the closure of $\mathbf{Q}\left(\zeta_{2 n}\right)$ in $k\left(\zeta_{2 n}\right)_{\mathfrak{p}}$ is an unramified extension of degree 2 , at most over $\mathbf{Q}_{p}$ and therefore $k\left(\zeta_{2 n}\right)_{\mathfrak{p}}=k_{(p)}$. Hence the solubility of the equation $x^{n}=\epsilon$ in $k_{(p)}=k\left(\zeta_{2 n}\right)_{\mathfrak{p}}$ yields that $\mathfrak{p}$ is completely decomposable at $K / k\left(\zeta_{2 n}\right)$.

Conversely, we suppose $p+1 \equiv 0 \bmod 2 n$ and each prime ideal of $k\left(\zeta_{2 n}\right)$ lying above $p$ is completely decomposable at $K$. Let $\mathfrak{P}$ be a prime ideal of $K$ lying above $p$, and set $\mathfrak{p}=\mathfrak{P} \cap k\left(\zeta_{2 n}\right)$. Since $p \equiv-1 \bmod 2 n$, the closure of $\mathbf{Q}\left(\zeta_{2 n}\right)$ in $K_{\mathfrak{P}}$ is an unramified extension of degree 2 over $\mathbf{Q}_{p}$ and so is the closure of $k$. Hence by the assumption, $K_{\mathfrak{P}}=k\left(\zeta_{2 n}\right)_{\mathfrak{p}}$ holds where $\mathfrak{p}=\mathfrak{P} \cap k\left(\zeta_{2 n}\right)$, and the equation $x^{n}=\epsilon$ is completely soluble over $k\left(\zeta_{2 n}\right)_{\mathfrak{p}}=k_{(p)}$. Take a generator $\alpha \in \mathfrak{o}_{k}$ of $\left(\mathfrak{o}_{k} /(p)\right)^{\times}$and an integer $u$ such that $\left(\alpha^{u}\right)^{n} \equiv \epsilon \bmod (p) .1 \equiv \epsilon^{r} \equiv \alpha^{\text {run }} \bmod (p)$ implies run $\equiv$ $0 \bmod p^{2}-1$ and for any proper divisor $t$ of $r$, tun $\not \equiv 0 \bmod p^{2}-1$ holds from the definition of $r$. Set $r u n=w\left(p^{2}-1\right)(w \in \mathbf{Z})$; then $(r, w)=1$ holds. If a prime number $q$ divides $(r, p-1)$, then $\frac{2(p+1)}{r}=\frac{I_{p}}{(p-1) / 2} \in \mathbf{Z}$ implies $q \mid 2(p+1)$ by $q \mid r$ and hence $q=2$ because of $q \mid(p-1)$. Set $r=2^{t} \cdot r^{\prime}\left(r^{\prime}\right.$ : odd); then we have shown $\left(r^{\prime}, p-1\right)=1$ and then $r u=2^{t} \cdot r^{\prime} u=w(p-1) \frac{p+1}{n}$ and $(r, w)=1=\left(r^{\prime}, p-1\right)$ imply $\left(r^{\prime}, w(p-1)\right)=1$ and $2^{t} u=w(p-1) \frac{p+1}{n r^{\prime}}$ and

$$
n r^{\prime} \mid(p+1)
$$

To show $n \frac{p-1}{2} \mid I_{p}$, i.e., $\frac{I_{p}}{n(p-1) / 2}=\frac{2(p+1)}{n r} \in \mathbf{Z}$, we have only to show $\operatorname{ord}_{2} \frac{2(p+1)}{n r} \geq 0$, where $a:=\operatorname{ord}_{2}(b)$ is defined by $2^{a} \| b$. At the begining of the proof, we showed $4 \mid r$ and then $t \geq 2$. $(r, w)=1$ implies that $w$ is odd. 
If $n$ is odd, then $\frac{2(p+1)}{r}=\frac{I_{p}}{(p-1) / 2} \in \mathbf{Z}$ yields $\operatorname{ord}_{2} \frac{2(p+1)}{n r}=\operatorname{ord}_{2} \frac{2(p+1)}{r} \geq 0$. If $n$ is even, then $p+1 \equiv 0 \bmod 4$ implies $0 \leq \operatorname{ord}_{2} u=\operatorname{ord}_{2} \frac{w\left(p^{2}-1\right)}{n r}=$ $\operatorname{ord}_{2} \frac{p+1}{n r}+\operatorname{ord}_{2}(p-1)=\operatorname{ord}_{2} \frac{p+1}{n r}+1=\operatorname{ord}_{2} \frac{2(p+1)}{n r}$. Thus we have shown $\operatorname{ord}_{2} \frac{2(p+1)}{n r} \geq 0$ and then $\frac{I_{p}}{n(p-1) / 2}=\frac{2(p+1)}{n r}$ is an integer and hence we have completed the proof of Lemma 3 .

THEOREM 2. Suppose that $N_{k / \mathbf{Q}}(\epsilon)=-1$ and $p$ is an odd prime number which remains prime in $k$. Let $n$ be a square-free natural number. Then $n \frac{p-1}{2} \mid I_{p}$ holds if and only if $k \not \subset \mathbf{Q}\left(\zeta_{2 n}\right), n$ is odd and for each prime ideal $\mathfrak{P}$ of $K=k\left(\zeta_{2 n}, \sqrt[n]{\epsilon}\right)$ lying above $p$, the Frobenius automorphism $\rho_{0}=\left(\frac{K / \mathbf{Q}}{\mathfrak{P}}\right)$ is equal to $\rho$ given in Proposition 1.

Proof. Suppose $n \frac{p-1}{2} \mid I_{p}$; then by Lemma 3, $\rho_{0}$ induces the complex conjugation on $\mathbf{Q}\left(\zeta_{2 n}\right)$ and the order of $\rho_{0}$ is two, since $K_{\mathfrak{P}}$ is a quadratic unramified extension of $\mathbf{Q}_{p}$. If $k \subset \mathbf{Q}\left(\zeta_{2 n}\right)$, then $\rho_{0}$ induces the trivial automorphism on $k$, which is a contradiction. Hence $k \not \subset \mathbf{Q}\left(\zeta_{2 n}\right)$ holds and then Proposition 1 implies that $n$ is odd and the uniqueness implies $\rho_{0}=\rho$.

Now let us show the converse. Since $\rho_{0}=\rho$ and $\rho$ induces the complex conjugation on $\mathbf{Q}\left(\zeta_{2 n}\right), p+1 \equiv 0 \bmod 2 n$ holds. Since the order of $\rho_{0}=2$, and the closure of $k$ in $K_{\mathfrak{P}}$ is a quadratic unramified extension of $\mathbf{Q}_{p}$, the relative degree of $\mathfrak{P}$ at $K / k$ and hence $K / k\left(\zeta_{2 n}\right)$ is one and then Lemma 3 implies $n \frac{p-1}{2} \mid I_{p}$.

\section{$\S 3$. Analytic part of the proof of the theorem}

Hereafter $q$ denotes a prime number and $p$ denotes an odd prime number which remains prime in the real quadratic field $k$. We set $\tilde{\ell}_{p}:=I_{p} / \ell_{p}$, which is an integer $([\mathrm{IK}])$. As in the Section 1 , we denote by $\mathbb{P}(x)$ the set of odd prime numbers $p \leq x$ which remains prime in $k$. Set for $x \geq 3$,

$$
\begin{aligned}
& N(x):=\sharp\left\{p \in \mathbb{P}(x) \mid \tilde{\ell}_{p}=1\right\}, \\
& N(x, \eta):=\sharp\left\{p \in \mathbb{P}(x) \mid q \nmid \tilde{\ell}_{p} \text { for }{ }^{\forall} q \leq \eta\right\}, \\
& M\left(x, \eta_{1}, \eta_{2}\right):=\sharp\left\{p \in \mathbb{P}(x)|q| \tilde{\ell}_{p} \text { for } \eta_{1}<{ }^{\exists} q \leq \eta_{2}\right\}, \\
& P(x, n):=\sharp\left\{p \in \mathbb{P}(x)|n| \tilde{\ell}_{p}\right\}, \\
& \xi_{1}:=6^{-1} \log x, \quad \xi_{2}:=\sqrt{x}(\log x)^{-2}, \quad \xi_{3}:=\sqrt{x} \log x .
\end{aligned}
$$

Then it is easy to see $N\left(x, \xi_{1}\right)-M\left(x, \xi_{1}, \xi_{2}\right)-M\left(x, \xi_{2}, \xi_{3}\right)-M\left(x, \xi_{3}, x-1\right) \leq$ $N(x)=N(x, x-1) \leq N\left(x, \xi_{1}\right)$. 
LEMMA 1. $M\left(x, \xi_{2}, \xi_{3}\right)=O\left((x \log (\log x)) /(\log x)^{2}\right)$.

Proof. Since $\tilde{\ell}_{p}=I_{p} / \ell_{p}$ divides $2 I_{p} /(p-1)$ and $I_{p}$ divides $p^{2}-1, \tilde{\ell}_{p}$ divides $2(p+1)$. For a prime number $q$ with $\xi_{2}<q \leq \xi_{3}, q \mid \tilde{\ell}_{p}$ implies $q \mid 2(p+1)$ and then $p \equiv-1 \bmod q$. Thus we have

$$
\begin{aligned}
M\left(x, \xi_{2}, \xi_{3}\right) & \leq \sum_{\xi_{2}<q \leq \xi_{3}} \sharp\{p \in \mathbb{P}(x) \mid p \equiv-1 \bmod q\} \\
& =O\left((x \log (\log x)) /(\log x)^{2}\right),
\end{aligned}
$$

as is shown in $[\mathrm{H}]$.

LEMMA 2. $M\left(x, \xi_{3}, x-1\right)=O\left(x(\log x)^{-2}\right)$.

Proof. For a prime number $p \in \mathbb{P}(x)$, suppose that a prime number $q$ with $\xi_{3}<q \leq x-1$ satisfies $q \mid \tilde{\ell}_{p}$. Set

$$
\delta= \begin{cases}1, & \text { if } N_{k / \mathbf{Q}}(\epsilon)=1 \\ 2, & \text { if } N_{k / \mathbf{Q}}(\epsilon)=-1\end{cases}
$$

Then $\delta \ell_{p}=p-1$ and $\frac{2(p+1)}{q \sharp E((p))}=\frac{2(p+1) I_{p}}{q \sharp E((p)) I_{p}}=\frac{2(p+1) \ell_{p} \tilde{\ell}_{p}}{q\left(p^{2}-1\right)}=\frac{2 \tilde{\ell}_{p}}{\delta q}$ is an integer. Hence $\sharp E((p))$ divides $2(p+1) / q$, where we note that $2(p+1) / q$ is an even integer. Thus $\epsilon^{2(p+1) / q} \equiv 1 \bmod (p)$ holds and then $N_{k / \mathbf{Q}}\left(\epsilon^{2(p+1) / q}-\right.$ $1) \equiv 0 \bmod p^{2}$. Here $2(p+1) / q<2(x+1) / \sqrt{x} \log x \ll \sqrt{x} / \log x$. Thus $p^{2}$ divides $\prod_{m \ll \sqrt{x} / \log x, m \text { :even }} N_{k / \mathbf{Q}}\left(\epsilon^{m}-1\right)$. Denote by $\epsilon_{1}$ the conjugate of $\epsilon$; then $\left|\epsilon_{1}\right|<1$ and for an even integer $m$, we have $\left|N_{k / \mathbf{Q}}\left(\epsilon^{m}-1\right)\right|=$ $\left|\epsilon^{m}-1\right|\left|\epsilon_{1}^{m}-1\right|<\epsilon^{m}-1<\epsilon^{m}$, and then we have

$$
2^{2 M\left(x, \xi_{3}, x-1\right)} \leq \prod_{p} p^{2} \leq \prod_{m \ll \sqrt{x} / \log x} \epsilon^{m},
$$

where $p$ runs over the set which defines $M\left(x, \xi_{3}, x-1\right)$. Therefore we have $M\left(x, \xi_{3}, x-1\right) \ll \sum_{m<\sqrt{x} / \log x} m \ll x /(\log x)^{2}$.

LEMmA 3. $M\left(x, \xi_{1}, \xi_{2}\right) \leq \sum_{\xi_{1}<q \leq \xi_{2}} P(x, q)$.

Proof. It is obvious.

LEMma 4. Set $Q\left(\xi_{1}\right):=\prod_{q \leq \xi_{1}} q$; then we have $N\left(x, \xi_{1}\right)=\sum_{n \mid Q\left(\xi_{1}\right)}$ $\mu(n) P(x, n)$, where $\mu(n)$ is the Möbius function. 
Proof. $N\left(x, \xi_{1}\right)$ is equal to

$$
\begin{aligned}
\sharp\{p & \left.\in \mathbb{P}(x) \mid\left(Q\left(\xi_{1}\right), \tilde{\ell}_{p}\right)=1\right\}=\sum_{p \in \mathbb{P}(x)} \sum_{n \mid\left(Q\left(\xi_{1}\right), \tilde{\ell}_{p}\right)} \mu(n) \\
& =\sum_{n \mid Q\left(\xi_{1}\right)} \mu(n) \sum_{p \in \mathbb{P}(x), n \mid \tilde{\ell}_{p}} 1=\sum_{n \mid Q\left(\xi_{1}\right)} \mu(n) P(x, n) .
\end{aligned}
$$

Thus we have

$$
N(x)=\sum_{n \mid Q\left(\xi_{1}\right)} \mu(n) P(x, n)+O\left(\sum_{\xi_{1}<q \leq \xi_{2}} P(x, q)\right)+O\left(x \log (\log x) /(\log x)^{2}\right) .
$$

Now let $n$ be a square-free natural number, and set

$$
K_{n}:= \begin{cases}k\left(\zeta_{2 n}, \sqrt[2 n]{\epsilon}\right), & \text { if } N_{k / \mathbf{Q}}(\epsilon)=1 \\ k\left(\zeta_{2 n}, \sqrt[n]{\epsilon}\right), & \text { if } N_{k / \mathbf{Q}}(\epsilon)=-1\end{cases}
$$

Then from Lemma 1 in the Section 2 follows that under the condition $k \not \subset \mathbf{Q}\left(\zeta_{2 n}\right)$

$$
\left[K_{n}: \mathbf{Q}\right]= \begin{cases}4 n \varphi(2 n), & \text { if } N_{k / \mathbf{Q}}(\epsilon)=1 \text { and } \sqrt{\epsilon} \notin k\left(\zeta_{2 n}\right), \\ 2 n \varphi(2 n), & \text { if } N_{k / \mathbf{Q}}(\epsilon)=1 \text { and } \sqrt{\epsilon} \in k\left(\zeta_{2 n}\right), \\ 2 n \varphi(n), & \text { if } N_{k / \mathbf{Q}}(\epsilon)=-1 \text { and } 2 \nmid n .\end{cases}
$$

Let $C$ be a union of conjugacy classes consisting of automorphisms $\rho$ in $\operatorname{Gal}\left(K_{n} / \mathbf{Q}\right)$ in Proposition 1 of the Section 2; then we have under the condition $k \not \subset \mathbf{Q}\left(\zeta_{2 n}\right)$

$$
\sharp(C)= \begin{cases}2, & \text { if } N_{k / \mathbf{Q}}(\epsilon)=1, \text { and } \\ & \text { either } 2 \mid n, \sqrt{\epsilon} \in k\left(\zeta_{2 n}\right) \text { and } \eta(\sqrt{\epsilon}) \sqrt{\epsilon}=1, \text { or } \sqrt{\epsilon} \notin k\left(\zeta_{2 n}\right), \\ 1, & \text { either if } N_{k / \mathbf{Q}}(\epsilon)=1,2 \nmid n, \text { and } \sqrt{\epsilon} \in k\left(\zeta_{2 n}\right), \\ & \text { or if } N_{k / \mathbf{Q}}(\epsilon)=-1 \text { and } 2 \nmid n, \\ 0, & \text { otherwise, }\end{cases}
$$

where $\eta$ is an automorphism such that it is the complex conjugation on $\mathbf{Q}\left(\zeta_{2 n}\right)$ and is the non-trivial automorphism on $k$, and note that the equality $\left[K_{n}: k\left(\zeta_{2 n}\right)\right]=n$ implies $\sqrt{\epsilon} \in k\left(\zeta_{2 n}\right)$ by Lemma 1 in the Section 2 when $N_{k / \mathbf{Q}}(\epsilon)=1$. Moreover Theorems 1 and 2 imply that

$$
P(x, n)= \begin{cases}\sharp\left\{p \in \mathbb{P}(x) \mid k \not \subset \mathbf{Q}\left(\zeta_{2 n}\right),\left(\frac{K_{n} / \mathbf{Q}}{\mathfrak{P}}\right) \in C\right\}, & \text { if } N_{k / \mathbf{Q}}(\epsilon)=1, \\ \sharp\left\{p \in \mathbb{P}(x) \mid k \not \subset \mathbf{Q}\left(\zeta_{2 n}\right), n \text { :odd, }\left(\frac{K_{n} / \mathbf{Q}}{\mathfrak{P}}\right) \in C\right\}, & \text { if } N_{k / \mathbf{Q}}(\epsilon)=-1,\end{cases}
$$


where $\mathfrak{P}$ is any prime ideal of $K_{n}$ lying above a prime ideal $p$. We apply the Chebotarev density theorem under the GRH ([LO], Théorème 4 in [S]):

Chebotarev Density Theorem. Suppose that the GRH holds for $K_{n}$. Let $C$ be the union of conjugacy classes of $\operatorname{Gal}\left(K_{n} / \mathbf{Q}\right)$ defined above. Denote by $\pi_{C}\left(x, K_{n}\right)$ the number of unramified prime number $p$ such that $\left(\frac{K_{n} / \mathbf{Q}}{\mathfrak{P}}\right) \in C$ and $p \leq x$, where $\mathfrak{P}$ is a prime ideal of $K_{n}$ lying above $p$. Then we have

$$
\left|\pi_{C}\left(x, K_{n}\right)-\frac{\sharp(C)}{\left[K_{n}: \mathbf{Q}\right]} \operatorname{Li}(x)\right|<c\left(\frac{\sharp(C)}{\left[K_{n}: \mathbf{Q}\right]} \sqrt{x} \log \left(d K_{n} x^{\left[K_{n}: \mathbf{Q}\right]}\right)\right),
$$

where $c$ is an absolute constant and $d K_{n}$ stands for the absolute discriminant of $K_{n}$.

Hereafter we apply this theorem assuming the GRH. Now set $d(n):=$ $\sharp(C)\left[K_{n}: \mathbf{Q}\right]^{-1}$; then we have

$$
d(n)= \begin{cases}(2 n \varphi(n))^{-1}, & \text { if } N_{k / \mathbf{Q}}(\epsilon)=-1, n \text { is odd and } k \not \subset \mathbf{Q}\left(\zeta_{n}\right), \\ (n \varphi(2 n))^{-1}, & \text { if } N_{k / \mathbf{Q}}(\epsilon)=1, n \text { is even, } \sqrt{\epsilon} \in k\left(\zeta_{2 n}\right), \\ & \eta(\sqrt{\epsilon}) \sqrt{\epsilon}=1 \text { and } k \not \subset \mathbf{Q}\left(\zeta_{2 n}\right), \\ (2 n \varphi(n))^{-1}, & \text { if } N_{k / \mathbf{Q}}(\epsilon)=1, n \text { is odd, } \sqrt{\epsilon} \in k\left(\zeta_{2 n}\right) \text { and } \\ & k \not \subset \mathbf{Q}\left(\zeta_{2 n}\right), \\ (2 n \varphi(2 n))^{-1}, & \text { if } N_{k / \mathbf{Q}}(\epsilon)=1, \sqrt{\epsilon} \notin k\left(\zeta_{2 n}\right) \text { and } k \not \subset \mathbf{Q}\left(\zeta_{2 n}\right), \\ 0, & \text { otherwise. }\end{cases}
$$

Note that $d(n)=0$ if $k \subset \mathbf{Q}\left(\zeta_{2 n}\right)$. By the theory of algebraic number fields, it is easy to see

$$
d K_{n} \mid(2 n)^{8 n \varphi(2 n)} D_{0}^{2 n \varphi(2 n)}
$$

where $D_{0}$ is the discriminant of $k$ as in the introduction. Theorems 1 and 2 in the Section 2 imply $\pi_{C}\left(x, K_{n}\right)=P(x, n)$ under the following condition $c(n)$ :

$$
\begin{cases}k \not \subset \mathbf{Q}\left(\zeta_{2 n}\right), & \text { if } N_{k / \mathbf{Q}}(\epsilon)=1, \\ n: \text { odd and } k \not \subset \mathbf{Q}\left(\zeta_{2 n}\right), & \text { if } N_{k / \mathbf{Q}}(\epsilon)=-1 .\end{cases}
$$

Note that if the condition $c(n)$ is not satisfied, then $d(n)=0$ holds. Hence we have

$$
\begin{gathered}
N(x)=\sum_{\substack{n \mid Q\left(\xi_{1}\right) \\
c(n)}} \mu(n) \pi_{C}\left(x, K_{n}\right)+O\left(\sum_{\substack{\xi_{1}<q \leq \xi_{2} \\
c(q)}} \pi_{C}\left(x, K_{q}\right)\right) \\
\quad+O\left(x \log (\log x) /(\log x)^{2}\right) .
\end{gathered}
$$


LEMMA 5. $\quad \sum_{\xi_{1}<q \leq \xi_{2}, c(q)} \pi_{C}\left(x, K_{q}\right)=O\left(x \log (\log x) /(\log x)^{2}\right)$.

Proof. It is easy to see that

$$
\begin{aligned}
& \sum_{\substack{\xi_{1}<q \leq \xi_{2} \\
c(q)}} \pi_{C}\left(x, K_{q}\right) \\
& \quad=\sum_{\xi_{1}<q \leq \xi_{2}} d(q) \operatorname{Li}(x)+O\left(\sum_{\xi_{1}<q \leq \xi_{2}} d(q) \sqrt{x} \log \left(d K_{q} x^{\left[K_{q}: \mathbf{Q}\right]}\right)\right) .
\end{aligned}
$$

Now we have

$$
\sum_{\xi_{1}<q \leq \xi_{2}} d(q) \ll \sum_{q>6^{-1} \log x} q^{-2} \ll 1 / \log x
$$

and

$$
\sum_{\xi_{1}<q \leq \xi_{2}} d(q) \log d K_{q} \ll \sum_{\xi_{1}<q \leq \xi_{2}}(\log q+1) \ll \sqrt{x}(\log x)^{-2}<\sqrt{x} \frac{\log \log x}{(\log x)^{2}}
$$

and lastly

$$
\begin{aligned}
\sum_{\xi_{1}<q \leq \xi_{2}} d(q)\left[K_{q}: \mathbf{Q}\right] \log x & \ll \pi\left(\xi_{2}\right) \log x \ll \sqrt{x}(\log x)^{-2} \\
& \ll \sqrt{x} \log \log x /(\log x)^{2} .
\end{aligned}
$$

From these follows the assertion.

LEMMA 6.

$$
\sum_{\substack{n \mid Q\left(\xi_{1}\right) \\ c(n)}} \mu(n) \pi_{C}\left(x, K_{n}\right)=\left(\sum_{n=1}^{\infty} \mu(n) d(n)\right) \operatorname{Li}(x)+O\left(x \log \log x /(\log x)^{2}\right) .
$$

Proof. By using the Chebotarev density theorem under the GRH, we have

the left-hand side

$$
\begin{aligned}
& =\sum_{n \mid Q\left(\xi_{1}\right)} \mu(n)\left\{d(n) \operatorname{Li}(x)+O\left(d(n) \sqrt{x} \log \left(d K_{n} x^{\left[K_{n}: \mathbf{Q}\right]}\right)\right)\right\} \\
& =\left(\sum_{n \mid Q\left(\xi_{1}\right)} \mu(n) d(n)\right) \operatorname{Li}(x)+\sqrt{x} \log x O\left(\sum_{n \mid Q\left(\xi_{1}\right)} d(n)\left[K_{n}: \mathbf{Q}\right]\right) \\
& \quad+\sqrt{x} O\left(\sum_{n \mid Q\left(\xi_{1}\right)} d(n) \log d K_{n}\right) .
\end{aligned}
$$


The first term is equal to

$$
\sum_{n \geq 1} \mu(n) d(n)+O\left(\sum_{n}^{\star}(n \varphi(n))^{-1}\right)
$$

where $\sum_{n}^{\star}$ means that the sum on $n$ which has a prime divisor larger than $\xi_{1}=6^{-1} \log x$, and it is easy to see

$$
\begin{aligned}
& \sum_{n}^{\star}(n \varphi(n))^{-1}<\sum_{n>\xi_{1}} \frac{1}{n^{2}} \frac{n}{\varphi(n)} \\
& \ll \sum_{n>\xi_{1}} n^{-2} \sum_{d \mid n} 1 / d=\sum_{d=1}^{\infty} 1 / d \sum_{m>\xi_{1} / d}^{\infty}(m d)^{-2} \\
& \ll \sum_{d=1}^{\infty} 1 / d^{3} \cdot \frac{1}{\xi_{1} / d} \ll 1 / \xi_{1} \ll 1 / \log x .
\end{aligned}
$$

We note that $\log Q\left(\xi_{1}\right) /\left(6^{-1} \log x\right)=\sum_{q \leq 6^{-1}} \log x \log q /\left(6^{-1} \log x\right)<1.1$ and then $Q\left(\xi_{1}\right)<x^{1.1 / 6}$ if $x$ is large. The second term is

$$
\begin{aligned}
\sqrt{x} \log x O\left(\sum_{n \mid Q\left(\xi_{1}\right)} 1\right) & =\sqrt{x} \log x O\left(Q\left(\xi_{1}\right)^{\delta}\right) \\
& =O\left(x \log \log x /(\log x)^{2}\right)
\end{aligned}
$$

where $\delta$ is an arbitrary small positive number. The third term is

$$
\begin{aligned}
\sqrt{x} & O\left(\sum_{n \mid Q\left(\xi_{1}\right)}\left(\frac{n \varphi(n)}{n \varphi(n)} \log n+1\right)\right)=\sqrt{x} O\left(\sum_{n<Q\left(\xi_{1}\right)}(\log n+1)\right) \\
& =O\left(\sqrt{x} Q\left(\xi_{1}\right) \log Q\left(\xi_{1}\right)\right)=\sqrt{x} O\left(x^{1.1 / 6} \log x^{1.1 / 6}\right) \\
& =O\left(x \log \log x /(\log x)^{2}\right) .
\end{aligned}
$$

Thus we have

$$
N(x)=\left(\sum_{n=1}^{\infty} \mu(n) d(n)\right) \operatorname{Li}(x)+O\left(x \log (\log x) /(\log x)^{2}\right)
$$

and we have only to show that the infinite series $\sum_{n=1}^{\infty} \mu(n) d(n)$ is a positive constant to complete the proof of the main theorem. The absolute convergence follows from $\varphi(n) \gg n / \log \log n$ and then $\left|\sum_{n=1}^{\infty} \mu(n) d(n)\right| \ll$ $\sum_{n=1}^{\infty}(\log \log n) / n^{2}<\infty$. Set $c_{0}:=\sum_{n=1}^{\infty} \mu(n) d(n)$. 
(I) The case of $N_{k / \mathbf{Q}}(\epsilon)=-1$.

In this case, we have $c_{0}=\sum_{n=1, n \text { :odd, } D_{0} \nmid n}^{\infty} \mu(n) d(n)$, where $D_{0}$ is the discriminant of $k$.

(I.1) The case of $D_{0} \equiv 0 \bmod 2$.

We have

$$
c_{0}=\sum_{\substack{n=1 \\ n: \text { odd }}}^{\infty} \frac{\mu(n)}{2 n \varphi(n)}=\prod_{q}\left(1-\frac{1}{q(q-1)}\right)=A>0,
$$

where $q$ runs over the set of all prime numbers.

(I.2) The case of $D_{0} \equiv 1 \bmod 2$.

In this case, we have

$$
\begin{aligned}
c_{0} & =\sum_{\substack{n=1, n \text { :odd } \\
D_{0} \nmid n}}^{\infty} \frac{\mu(n)}{2 n \varphi(n)}=\sum_{n=1, n \text { odd }}^{\infty} \frac{\mu(n)}{2 n \varphi(n)}-\sum_{\substack{n=1, n: \text { odd } \\
D_{0} \mid n}}^{\infty} \frac{\mu(n)}{2 n \varphi(n)} \\
& =A-\sum_{\substack{m=1, m: \text { odd } \\
\left(m, D_{0}\right)=1}}^{\infty} \frac{\mu\left(D_{0}\right) \mu(m)}{2 D_{0} m \varphi\left(D_{0} m\right)} \\
& =A-\frac{\mu\left(D_{0}\right)}{2 D_{0} \varphi\left(D_{0}\right)} \sum_{m=1, m: \text { odd }}^{\left(m, D_{0}\right)=1} \frac{\mu(m)}{m \varphi(m)} \\
& =A-\frac{\mu\left(D_{0}\right)}{2 D_{0} \varphi\left(D_{0}\right)} \prod_{q \nmid 2 D_{0}}\left(1-\frac{1}{q(q-1)}\right) \\
& =A\left\{1-\frac{\mu\left(D_{0}\right)}{2 D_{0} \varphi\left(D_{0}\right)} \prod_{q \mid 2 D_{0}} \frac{q(q-1)}{q^{2}-q-1}\right\} \\
& =A\left(1-\mu\left(D_{0}\right) \prod_{q \mid D_{0}} \frac{1}{q^{2}-q-1}\right)>0 .
\end{aligned}
$$

(II) The case of $N_{k / \mathbf{Q}}(\epsilon)=1$.

We note some facts.

- Suppose that $m$ is odd square-free. $k \subset \mathbf{Q}\left(\zeta_{2 m}\right)=\mathbf{Q}\left(\zeta_{m}\right)$ if and only if $D_{0} \mid m . k \subset \mathbf{Q}\left(\zeta_{4 m}\right)$ if and only if $D_{0} \mid 4 m$.

- $\sum_{m=1, m: \text { odd }}^{\infty} \mu(m) / m \varphi(m)=2 A$. 
- $\sum_{m \text { :odd, square-free }} 1 / m \varphi(m)=\prod_{2 \nmid p}\left(1+\frac{1}{p(p-1)}\right)=1.2957 \cdots$.

Now we have

$$
\begin{aligned}
& c_{0}=\sum_{n=1}^{\infty} \mu(n) d(n)
\end{aligned}
$$

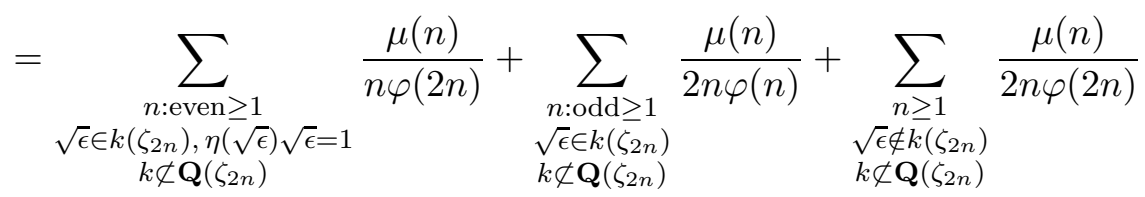

$$
\begin{aligned}
& =-\frac{1}{4} \sum_{\substack{m: \text { odd } \geq 1 \\
\sqrt{\epsilon} \in k\left(\zeta_{4 m}\right), \eta(\sqrt{\epsilon}) \sqrt{\epsilon}=1 \\
k \not \subset \mathbf{Q}\left(\zeta_{4 m}\right)}} \frac{\mu(m)}{m \varphi(m)}+\frac{1}{2} \sum_{\substack{m: \text { odd } \geq 1 \\
\sqrt{\epsilon} \in k\left(\zeta_{2 m}\right) \\
k \not \subset \mathbf{Q}\left(\zeta_{2 m}\right)}} \frac{\mu(m)}{m \varphi(m)} \\
& +\frac{1}{2} \sum_{\substack{m: \text { odd } \geq 1 \\
\sqrt{\epsilon} \notin k\left(\zeta_{2 m}\right) \\
k \not \subset \mathbf{Q}\left(\zeta_{2 m}\right)}} \frac{\mu(m)}{m \varphi(m)}-\frac{1}{8} \sum_{\substack{m: \operatorname{odd} \geq 1 \\
\sqrt{\epsilon} \notin k\left(\zeta_{4 m}\right) \\
k \not \subset \mathbf{Q}\left(\zeta_{4 m}\right)}} \frac{\mu(m)}{m \varphi(m)} \\
& =\frac{1}{2} \sum_{\substack{m: \text { odd } \geq 1 \\
k \not \subset \mathbf{Q}\left(\zeta_{m}\right)}} \frac{\mu(m)}{m \varphi(m)}-\frac{1}{4} \sum_{\substack{m: \text { odd } \geq 1 \\
\sqrt{\epsilon} \in k\left(\zeta_{4 m}\right), \eta(\sqrt{\epsilon}) \sqrt{\epsilon}=1 \\
k \not \subset \mathbf{Q}\left(\zeta_{4 m}\right)}} \frac{\mu(m)}{m \varphi(m)} \\
& -\frac{1}{8} \sum_{\substack{m: \text { odd } \geq 1 \\
\sqrt{\epsilon} \notin k\left(\zeta_{4 m}\right) \\
k \not \subset \mathbf{Q}\left(\zeta_{4 m}\right)}} \frac{\mu(m)}{m \varphi(m)} .
\end{aligned}
$$

The absolute value of the sum of the second and third terms is less than

$$
\begin{aligned}
& \frac{1}{4} \sum_{\substack{m: \text { odd, square-free } \\
\sqrt{\epsilon} \in k\left(\zeta_{4 m}\right)}} \frac{1}{m \varphi(m)}+\frac{1}{8} \sum_{\substack{m: \text { odd, square-free } \\
\sqrt{\epsilon} \notin k\left(\zeta_{4 m}\right)}} \frac{1}{m \varphi(m)} \\
& =\frac{1}{8} \sum_{\substack{m: \text { odd, square-free } \\
\sqrt{\epsilon} \in k\left(\zeta_{4 m}\right)}} \frac{1}{m \varphi(m)}+\frac{1}{8} \sum_{m: \text { odd, square-free }} \frac{1}{m \varphi(m)} \\
& \leq \frac{1}{4} \sum_{m: \text { odd, square-free }} \frac{1}{m \varphi(m)}-\frac{1}{8} \\
& =0.1989 \cdots,
\end{aligned}
$$

where the last inequality follows from $\sqrt{\epsilon} \notin k\left(\zeta_{4}\right)$. 
If $4 \mid D_{0}$, then the first term is equal to

$$
\frac{1}{2} \sum_{m: \text { odd }} \frac{\mu(m)}{m \varphi(m)}=A=0.3739 \ldots
$$

and hence $c_{0}>0$ holds.

If $D_{0}$ is odd, then the first term is

$$
\begin{aligned}
& \frac{1}{2} \sum_{\substack{m: \text { odd } \\
D_{0} \nmid m}} \frac{\mu(m)}{m \varphi(m)}=\frac{1}{2} \sum_{m: \text { odd }} \frac{\mu(m)}{m \varphi(m)}-\frac{1}{2} \sum_{\substack{m: \text { odd } \\
D_{0} \mid m}} \frac{\mu(m)}{m \varphi(m)} \\
& \quad=A-\frac{\mu\left(D_{0}\right)}{2 D_{0} \varphi\left(D_{0}\right)} \sum_{\substack{n: \text { odd } \\
\left(n, D_{0}\right)=1}} \frac{\mu(n)}{n \varphi(n)} \\
& =A-\frac{\mu\left(D_{0}\right)}{2 D_{0} \varphi\left(D_{0}\right)} \prod_{q \nmid 2 D_{0}}\left(1-\frac{1}{q(q-1)}\right) \\
& =A\left(1-\frac{\mu\left(D_{0}\right)}{2 D_{0} \varphi\left(D_{0}\right)} \prod_{q \mid 2 D_{0}} \frac{q(q-1)}{q^{2}-q-1}\right) \\
& \quad=A\left(1-\mu\left(D_{0}\right) \prod_{q \mid 2 D_{0}} \frac{1}{q^{2}-q-1}\right) \\
& \geq A(1-1 / 19)=0.3542 \cdots
\end{aligned}
$$

where the inequality follows from the fact that $D_{0}$ is divisible by a prime $\geq 5$ and hence we have $c_{0}>0$. Thus we have completed the proof of the main theorem.

\section{REFERENCES}

[H] C. Hooley, On Artin's conjecture, J. reine angew. Math., 225 (1967), 209-220.

[IK] M. Ishikawa and Y. Kitaoka, On the distribution of units modulo prime ideals in real quadratic fields, J. reine angew. Math., 494 (1998), 65-72.

[LO] J. C. Lagarias and A. M. Odlyzko, Effective version of the Chebotarev density theorem, Algebraic Number Fields (Fröhlich, ed.), Academic Press (1977), 409-464.

$[\mathrm{M}]$ K. Masima, On the distribution of units in the residue class field of real quadratic fields and Artin's conjecture (in Japanese), RIMS Kokyuroku, 1026 (1998), 156-166.

[S] J. P. Serre, Quelques applications du théorème de densité de Chebotarev, I.H.E.S., 54 (1981), 323-401. 
Yen-Mei J. Chen

Dept. of Math.

Tamkang University

Tamshui, Taipei

Taiwan

ymjchen@mail.tku.edu.tw

Yoshiyuki Kitaoka

Dept. of Math.

Meijo University

Tenpaku-ku

Nagoya, 468-8502

Japan

kitaoka@meijo-u.ac.jp

Jing $\mathrm{Yu}$

Institute of Math.

Academia Sinica

Nakang, Taipei

Taiwan

and National Center for Theoretical Science

Hsinchu

Taiwan

yu@math.sinica.edu.tw 